\title{
Primary Cutaneous Plasmacytoma Treated with Palliative Radiotherapy: A Case Report and Literature Review
}

Suzanne Alkul, MD1, Yuangao Liu, BS², Scott Whipple, PA-C ${ }^{3}$,Gordana Verstovsek, MD ${ }^{4}$, Soo Jung Kim MD, PhD'

1 Department of Dermatology, Baylor College of Medicine, Houston, TX

2 Baylor College of Medicine, Houston, TX

3 Department of Dermatology, Michael E. DeBakey VAMC, Houston, TX

4 Department of Pathology and Immunology, Baylor College of Medicine, Houston, TX

\section{ABSTRACT}

Introduction: Primary cutaneous plasmacytomas (PCP) are very rare plasma cell neoplasms present in the skin or subcutaneous tissue without underlying multiple myeloma (MM).

Case Report: A 78-year-old man was referred to dermatology by wound care for a three-year history of non-healing, enlarging, bleeding lesions on the right lower extremity. Examination revealed multiple firm, tender, slightly mobile erythematous to violaceous nodules ranging from 1 to $5 \mathrm{~cm}$ in size, some with erosion and heme crusting. Histopathologic analysis of skin biopsies showed an expansile nodular plasmocytic infiltrate in the dermis. A diagnosis of PCP was made based on clinical and histopathological features. Our patient underwent palliative radiation and responded to the therapy with decrease in size of all lesions.

Conclusion: We present a case of PCP presenting as multiple erythematous nodules as opposed to a solitary neoplasm, which likely represents a spectrum of variation of one tumor. To diagnose PCP, patients should not meet criteria for MM. All patients with new solitary plasmacytoma should be evaluated with skeletal survey or computed tomography, MRI, and PET/CT to rule out additional lesions. PCPs are usually indolent but $10 \%$ to $30 \%$ progress to MM within 10 years. Radiation therapy is considered the standard treatment, and additional clinical trials are underway to test the role of adjuvant chemotherapies.

\section{INTRODUCTION}

Primary extramedullary plasmacytoma $(E M P)$ is a rare plasma cell neoplasm. About $80 \%$ of EMPs occur in the head and neck regions, gastrointestinal tract, and the lymph nodes ${ }^{1}$. Only about $2-4 \%$ EMPs present in the skin or subcutaneous tissue and are referred to as primary cutaneous plasmacytomas $(\mathrm{PCP})^{1}$. They are, by definition, isolated neoplasms and occur without underlying multiple myeloma (MM). We present a case of a PCP presenting as enlarging, erythematous nodules cared for by a wound care team before being referred to dermatology for assistance in diagnosis.

\section{CASE REPORT}

A 78-year-old man with a past medical history of congestive heart failure and endstage renal disease on hemodialysis was referred to dermatology for a three-year history of non-healing, enlarging, bleeding lesions on the right lower extremity. He denied constitutional symptoms including 
fatigue, weight loss, night sweats, fever, or chills. The nodules were previously treated by the wound care team with zinc oxide, silver impregnated gel, and petrolatum impregnated non-adherent dressings. Examination revealed multiple firm, tender, slightly mobile erythematous to violaceous nodules ranging from 1 to $5 \mathrm{~cm}$ in size, some with erosion and heme crusting (Figure 1).

Additionally, the lower legs were noted to be hyperpigmented with $2+$ pitting edema, consistent with chronic stasis dermatitis. He had no inguinal lymphadenopathy.

Histopathologic analysis of skin biopsies showed an expansile nodular plasmocytic infiltrate in the dermis. By immunohistochemistry (IHC), lesion cells were positive for CD138 (strong, diffuse), CD45 (partial), CD10 (partial), and epithelial membrane antigen (partial) (Figure 2). The following markers were negative: CD20, PAX5, CD30, cyclin D1, EBER-ISH, anaplastic lymphoma kinase-1, cytokeratin (AE1/AE3), and panmelanoma. Kappa and lambda dual stain was consistent with kappa predominance, and Ki67 showed a proliferation rate of approximately $50 \%$. The morphologic and IHC features support the diagnosis of plasmacytoma. Tissue cultures were negative for fungus and acid-fast bacteria.

A bone marrow aspirate and biopsy of the left posterior superior iliac spine revealed a normal percentage of plasma cells $(3 \%)$ with no evidence of a clonal plasma cell population. Positron emission tomography (PET) scan showed innumerable nodules of the right leg with the depth of involvement of the largest lesion ranged between 1.1 to 2.3 $\mathrm{cm}$ on the right lower leg without any signs of metastasis or bone involvement. Serum and urine protein electrophoresis were both negative. Although a nonspecific marker, Beta 2 microglobulins were elevated, light chain ratio and serum protein electrophoresis were within normal limits. Taken together, our patient did not meet the International Myeloma Working Group (IMWG) criteria for diagnosis of MM and had no evidence of underlying bone marrow involvement $^{2}$. Thus, a diagnosis of PCP was made based on clinical and histopathological features.

Due to his underlying comorbidities and questionable ability to recover from a definitive radiotherapy dose and possible complications including radiation dermatitis, he instead underwent palliative radiation with a dose of 2000cGY in 10 fractions over 2 weeks. The patient responded to the therapy with decrease in size of all the lesions. The patient died 6 months after diagnosis and palliative treatment of cardiac arrest unrelated to the diagnosis of PCP.

\section{DISCUSSION}

Plasma cell neoplasms can be classified into the following types: MM (bone marrow and other organ system involvement) and solitary plasmacytoma (SP) that can present either as EMP in soft tissues or as solitary bone plasmacytoma $(\mathrm{SBP})^{3,4}$. Most plasmacytomas are seen in the setting of MM $(94 \%)^{3,4}$, especially in advanced myeloma with poor prognosis ${ }^{5}$. SP is a localized clonal plasma cell infiltration either in the bone or extramedullary sites without systemic disease. To be considered SBP or EMP, bone marrow biopsy should demonstrate less than $10 \%$ plasma cells, and IMWG criteria should not be met. Otherwise, it would be classified as MM. 

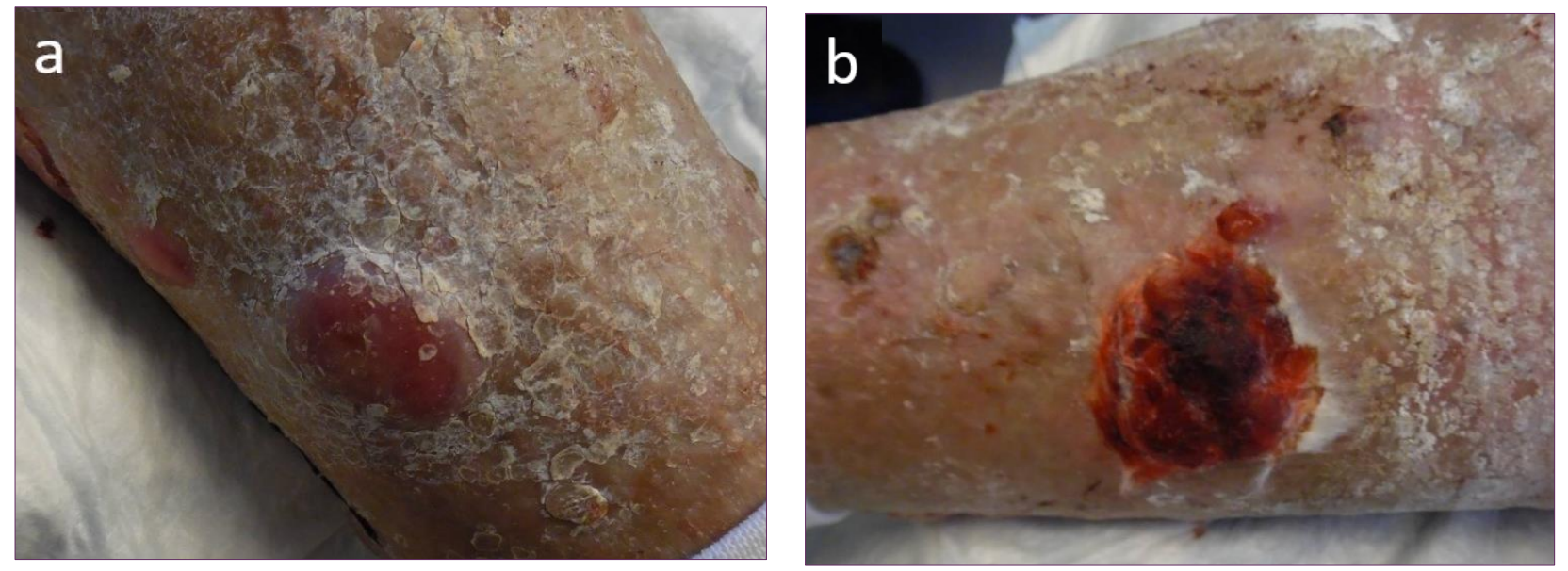

Figure 1: Right lower leg with erythematous to violaceous nodules A) and eroded nodule with heme crusting $\mathbf{B}$ ) on a background of stasis dermatitis and xerosis.

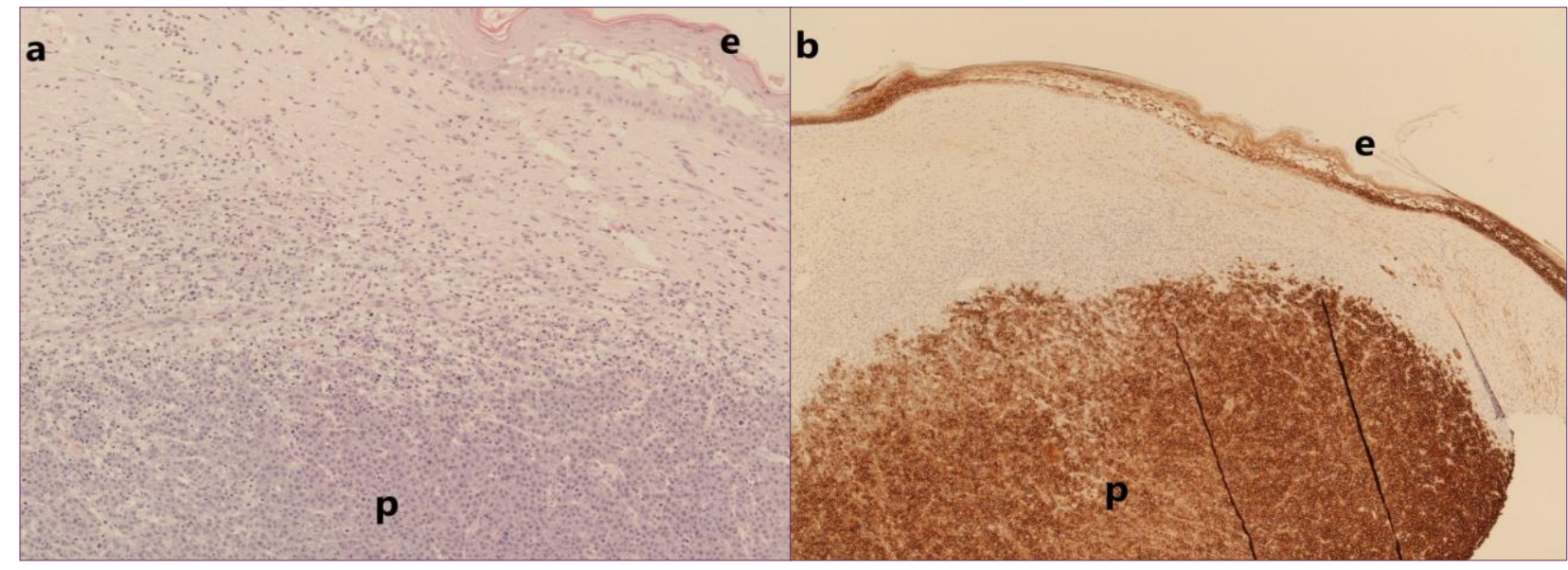

Figure 2: Skin biopsy shows expansile nodular plasmacytoid cell infiltrate in the deep dermis underlying epidermis E) 1A) Infiltrate is strongly positive for CD138 by immunohistochemistry confirming plasmacytoma P) 1B) (1A, Hematoxylin and Eosin 100x; 1B Immunoperoxidase 40x)

Primary cutaneous plasmacytomas are even more rare type of EMP confined to only skin without underlying systemic disease ${ }^{6}$.

Pathogenesis in some PCPs has been associated with immunosuppression and viral infections ${ }^{7}$. Clinically, cutaneous plasmacytomas manifests as indurated flesh colored, erythematous, or violaceous nodules or plaques and can be found anywhere on the body ${ }^{5,7}$. They can be solitary or multiple, with the reported prevalence of multiple lesions ranging from
38-44\% 6,8. Caers et al published recommendations for the diagnosis, treatment, and prognosis of $\mathrm{SPs}^{3}$. Diagnosis is made on the basis of a tissue biopsy with a diffuse dermal infiltrate of atypical and pleomorphic plasma cells, which are positive for CD138 and/or CD383. Monoclonality can be confirmed with kappa or lambda light chain restriction by polymerase chain reaction $(\mathrm{PCR}), \mathrm{IHC}$, or in situ hybridization. All patients presenting with a new SP should be evaluated with a skeletal survey or computed tomography (CT). Magnetic 
resonance imaging (MRI) and $\mathrm{PET} / \mathrm{CT}^{3}$ are also required to rule out additional lesions as well as MM. Although EMPs, including PCP, are usually indolent, $10 \%$ to $30 \%$ of EMPs progress to MM within 10 years ${ }^{3,4}$. Presence of clonal cell population in bone marrow and the presence of additional lesions on PET/CT are negative prognostic factors and should be included in the initial assessment. Patients with solitary lesions have a longer recurrence-free survival of 11 years compared to 1.4 years for patients with multiple lesions ${ }^{9}$. Furthermore, patients with multiple skin lesions have higher mortality from disseminated disease. Taken together, multiple lesions at the initial presentation are likely to be a predictor of poorer outcomes and subsequent disease progression $n^{6,9,10}$.

For treatment, radiation therapy is considered standard for $\mathrm{EMP}^{4}$. For local disease, as in PCP, surgery is also an option to resect large masses but should be followed by adjuvant radiotherapy ${ }^{3}$. For those patients presenting with multiple PCP lesions or persistent disease, additional benefit may be gained from systemic chemotherapy to prevent progression to $\mathrm{MM}^{9}$. Serum protein electrophoresis and immunofixation as well as the same imaging methods should be closely followed to assess the treatment response. Currently, additional clinical trials are underway to test the role of adjuvant chemotherapy including conventional chemotherapy, novel MM agents, immunomodulatory agents, and monoclonal antibodies ${ }^{3}$.

The distinction of PCP as a unique entity is a matter of debate. There are some who would categorize them as primary cutaneous marginal zone lymphomas (PCMZL) of mucosa-associated lymphoid tissue with plasma cell differentiation ${ }^{5,8}$ as they show clinical and immunologic phenotype overlap. In fact, the WHO-
EORTC classify PCPs with PCMZL ${ }^{11}$. As a distinguishing feature, the latter are generally CD20 positive and CD10 whereas the former express the opposite ${ }^{5,11}$. PCMZLs are generally low-grade malignancies with low rates of disease progression and recurrence; the disease specific 5-year survival approaches $100 \%{ }^{11}$. PCPs in contrast have the potential to progress, metastasize, and cause significant mortality, especially when multiple nodules are present, with up to $50 \%$ of reported patients developing systemic involvement and a median overall survival of 10.4 years ${ }^{9,12}$. Surgical excision and radiotherapy are standard treatment approach for both of these conditions ${ }^{9,11}$.

\section{CONCLUSION}

Plasma cell neoplasms encompass various clinical entities including MM, SBP, and EMP, with PCP involving the skin and soft tissue. PCP is a very rare entity and shows considerable clinical and phenotypic overlap with PMZCL. The present case describes multiple nodules as opposed to a solitary neoplasm, which likely represents a spectrum of variation of one tumor. Before SBP or EMP is considered, systemic involvement must be ruled out, and close follow-up is recommended to detect potential progression to MM.

\section{Conflict of Interest Disclosures: None}

Funding: None

Corresponding Author:

Suzanne Alkul, MD

1977 Butler Blvd Houston, TX 77030

Phone: 713-798-6131

Email: Alkul@bcm.edu

\section{References:}

1. Wiltshaw $\mathrm{E}$. The natural history of extramedullary plasmacytoma and its relation November 2021 Volume 5 Issue 6 
to solitary myeloma of bone and

myelomatosis. Med (United States).

1976;55(3):217-238. doi:10.1097/00005792-

197605000-00002

2. Rajkumar SV, Dimopoulos MA, Palumbo A, et al. International Myeloma Working Group updated criteria for the diagnosis of multiple myeloma. Lancet Oncol. 2014;15(12):e538e548. doi:10.1016/S1470-2045(14)70442-5

3. Caers J, Paiva B, Zamagni E, et al. Diagnosis, treatment, and response assessment in solitary plasmacytoma: Updated recommendations from a European Expert Panel. J Hematol Oncol. 2018;11(1):10. doi:10.1186/s13045-017-0549-1

4. Tsang RW, Campbell BA, Goda JS, et al. Radiation Therapy for Solitary Plasmacytoma and Multiple Myeloma: Guidelines From the International Lymphoma Radiation Oncology Group. Int J Radiat Oncol Biol Phys. 2018;101(4):794-808.

doi:10.1016/j.jrobp.2018.05.009

5. Rongioletti F, Patterson JW, Rebora A. The histological and pathogenetic spectrum of cutaneous disease in monoclonal gammopathies. J Cutan Pathol. 2008;35(8):705-721. doi:10.1111/j.16000560.2007.00884.x

6. Muscardin a LM, Pulsoni b A, Cerroni c L. Primary cutaneous plasmacytoma: Report of a case with review of the literature. J Am Acad Dermatol. 2000;43(5):962-965.

doi:10.1067/MJD.2000.103997

7. Walby ML, Ahluwalia J, Mehregan DR.

Primary cutaneous plasmacytoma in an HIVpositive patient. Int J Dermatol. 2016;55(4):464-467. doi:10.1111/j.13654632.2012.05764.x

8. Schmid U, Eckert F, Griesser H, et al. Cutaneous follicular lymphoid hyperplasia with monotypic plasma cells. A clinicopathologic study of 18 patients. Am J Surg Pathol. 1995;19(1):12-20. doi:10.1097/00000478199501000-00002

9. $\quad$ Tsang DS, Le LW, Kukreti V, Sun A. Treatment and outcomes for primary cutaneous extramedullary plasmacytoma: A case series. Curr Oncol. 2016;23(6):e630e646. doi:10.3747/co.23.3288

10. Miyamoto T, Kobayashi T, Hagari Y, Mihara $M$. The value of genotypic analysis in the assessment of cutaneous plasmacytomas. $\mathrm{Br}$ J Dermatol. 1997;137(3):418-421. doi:10.1046/J.1365-2133.1997.18611931.X

11. Willemze R, Jaffe ES, Burg G, et al. WHOEORTC classification for cutaneous lymphomas. Blood. 2005;105(10):3768-3785. doi:10.1182/BLOOD-2004-09-3502

12. Kazakov D V., Belousova IE, Müller B, et al. Primary cutaneous plasmacytoma: a clinicopathological study of two cases with a long-term follow-up and review of the literature. J Cutan Pathol. 2002;29(4):244248. doi:10.1034/J.1600-0560.2002.290408.X 